\title{
Access to dialysis services: A systematic mapping review based on geographical information systems
}

\author{
Benyamin Hoseini, ${ }^{1,2}$ Nasser Bagheri, ${ }^{3}$ Behzad Kiani, ${ }^{2}$ Amirabbas Azizi, ${ }^{4}$ Hamed Tabesh, ${ }^{2}$ \\ Mahmood Tara ${ }^{2}$ \\ ${ }^{1}$ Department of Health Information Technology, Neyshabur University of Medical Sciences, Neyshabur, \\ Iran; ${ }^{2}$ Department of Medical Informatics, Faculty of Medicine, Mashhad University of Medical Sciences, \\ Mashhad, Iran; ${ }^{3}$ Department of Health Services Research and Policy, College of Health and Medicine, \\ Biology and Environment, Australian National University, Canberra, ACT, Australia; ${ }^{4}$ Department of \\ Health Information Technology, School of Allied Health Professions, Ahvaz Jundishapur University of \\ Medical Sciences, Ahvaz, Iran
}

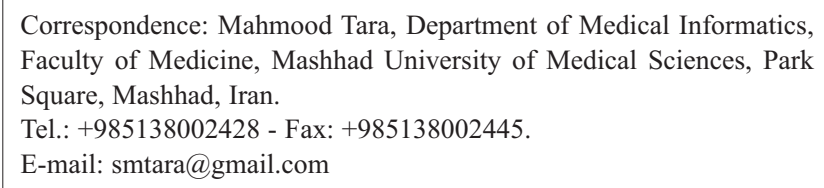

Key words: Access; Dialysis; Geographical information systems; Floating catchment; Renal.

Contributions: NB designed the study; BH searched for the literature. Then, the EndNote X5 (Thomson Reuters, New York, NY, USA) reference management software was applied to aggregate all the search strategy returns. Two reviewers (BH and $\mathrm{BK}$ ) screened the exported articles in EndNote and compared them with the inclusion and exclusion criteria independently. Any disagreement was resolved by another reviewer (MT), who was also responsible for the supervision of the research. Each study was assessed independently by two reviewers for the eligibility criteria. The full text of the qualified studies was retrieved and saved. The eligible papers were read, tagged and summarized by one reviewer $(\mathrm{BH})$ and then verified by the second reviewer (MT). Quality scores were assigned by two reviewers (BH and $\mathrm{AA}$ ) and verified by the third reviewer (HT). BH wrote the draft of the paper. $\mathrm{BK}$ and $\mathrm{BH}$ reviewed the paper and made revisions.

Acknowledgements: the authors would like to thank the authors of all the studies they reviewed.

Conflict of interest: the authors declare no potential conflict of interest.

Funding: the work was supported by a grant [941344] from Mashhad University of Medical Sciences Research Council.

Received for publication: 16 April 2017.

Revision received: 28 November 2017.

Accepted for publication: 28 November 2017.

(C) Copyright B. Hoseini et al., 2018

Licensee PAGEPress, Italy

Geospatial Health 2018; 13:577

doi: $10.4081 / \mathrm{gh} .2018 .577$

This article is distributed under the terms of the Creative Commons Attribution Noncommercial License (CC BY-NC 4.0) which permits any noncommercial use, distribution, and reproduction in any medium, provided the original author(s) and source are credited.

\begin{abstract}
Equitable access to healthcare services constitutes one of the leading priorities of healthcare provision and access to dialysis services (ADS) has an essential impact on patients depending on renal dialysis. The many existing GIS-based ADS evaluations include various spatial and non-spatial factors affecting ADS. We systematically mapped and reviewed the available literature with reference to this area identifying gaps in current GIS-based ADS measurements and developing recommendations for future studies. A threestep, systematic mapping review of the available GIS-related evidence in PubMed, Embase, Web of science, Scopus, Science Direct and IEEE Xplore was performed in May 2016 and the information collected updated October 2017 by two independent selection processes. The quality of the studies was assessed using an informal, mixed-approach scoring system. Out of 1119 literature references identified, 36 were identified and used for final review after removal of duplicates, study screenings and applying inclusion/exclusion criteria. Given the contents of the selected studies, three study groups were identified and 41 factors with potential effects on ADS determined. These studies mainly addressed the potential and/or spatial aspects of ADS. Our systematic mapping review of the evidence revealed that current GIS-based measures of ADS tend to calculate potential ADS instead of a realized one. It was also noted that listed factors affecting ADS were mainly nonspatial bringing forth the hypothesis that designing an integrated ADS index could possibly produce better ADS score than those currently advocated. Some primary and secondary research suggestions are made and a list of recommendations offered.
\end{abstract}

\section{Introduction}

Although equitable access to healthcare services (AHS) is a leading public health priority, the importance of AHS and the attention received from the policy managers differ from one disease to another. Access to dialysis services (ADS) is a crucial necessity for patients who have to travel to a dialysis facility three times a week (Mactier, 2007; Stephens et al., 2013). Thus, poorly developed ADS lead to poor health outcomes such as increased morbidity and mortality (Moist et al., 2008; Diamant et al., 2010; Rucker et al., 2011; Thompson et al., 2012).

A vital aspect of AHS is the ease with which they can be 
accessed and used when needed (McLafferty, 2003). AHS has five main dimensions: accessibility, availability, accommodation, affordability and acceptability (Levesque et al., 2013; Russell et al., 2013; Saurman, 2016). The accessibility and availability dimensions are usually related to geographical factors and are therefore labelled spatial accessibility (Mao and Nekorchuk, 2013), while non-geographic-dependent dimensions such as affordability, accommodation and acceptability are considered non-spatial (Guagliardo, 2004). Additionally, AHS can be divided into two broad categories: potential AHS and realized AHS. The former is simply defined as the presence of enabling healthcare, while the latter is the actual use of its services (Andersen, 1995).

Geographic information systems (GISs) enable researchers in the healthcare field to apply combined spatial accessibility measures for the inspection of the equitability of resource allocation. Various studies have demonstrated the application of GIS tools which are useful for calculation and visualization of accessibility scores (Guagliardo, 2004; Yang et al., 2006; Matsumoto et al., 2012; Kiani et al., 2017). Stephens et al. (2013) performed a GISbased measure of ADS which included travel impedance to dialysis facilities as an indicator. A recent study performed in Iran by Kiani et al. (2017) revealed the importance of spatial accessibility to dialysis services and showed that this variable is strongly underestimated when potential travel time is calculated (Kiani et al., 2017). They developed a comprehensive measure of revealed accessibility that includes travel time and some other spatial and also non-spatial factors into one indicator framework. However, their index does not include facility capacity. Matsumoto et al. (2012) designed an algorithm embedded in a GIS-based measure of ADS which demonstrates that patients cannot always be accepted by the nearest dialysis facility due to limited capacity. Another study performed in the US emphasized the acceptability dimension and revealed that dialysis patients may have a short travel time to one dialysis facility but might decide to go to another centre due to ethnic disparity (Saunders et al., 2014). Some studies, mainly conducted in Britain (Roderick et al., 1999; Christie et al., 2005; White et al., 2006; Judge et al., 2012), calculated deprivation as an important ADS indicator which includes some major non-spatial factors. Deprivation can also potentially affect need of dialysis services (NDS) (Roderick et al., 1999; Thomas, 2005; Yang et al., 2006; Judge et al., 2012). NDS is defined as the number of patients in an area who need dialysis (Yang et al., 2006).

The diversity of GIS-based ADS measures include various indicators with spatial as well as non-spatial confounding factors that may confuse researchers and policymakers. A mapping exercise could potentially lead to a more conclusive index producing better scores than currently used. GIS-based methods are inherently spatial; some of them, such as the two-step floating catchment area (2SFCA), demonstrated the capability of integrating both spatial and non-spatial AHS factors into one framework (Bagheri et al., 2008; McGrail and Humphreys, 2009; McGrail and Humphreys, 2015). Yang et al. (2006) provide a 2SFCA platform that integrates only dialysis patients and dialysis machines within a 30-minutes potential travel time catchment. Although this represents a certain progress, to the best of our knowledge, no study so far has made an attempt to develop a truly integrated GIS-based ADS index. In an effort to do so, we decided to focus on the gaps in the current approach to GIS-based ADS measures through a systematic review of the available literature. In addition, we aimed to develop recommendations and a list of factors affecting ADS that would improve the research on the measuring ADS based on GIS.

\section{Materials and Methods}

A systematic mapping review of the available GIS-based literature was performed. It aimed to describe the extent of the study on a particular topic and to identify knowledge gaps in the study base, where further primary and/or secondary studies are needed (Grant and Booth, 2009).

\section{Search strategy}

The scientific literature was explored with regard to relevant communications on ADS and the use of GIS. It was done in May 2016 and the information collected updated in October 2017. We included the following electronic databases: PubMed, Web of Science, Scopus, ScienceDirect, EMBASE and IEEE Xplore. Initially, the databases were systematically searched using a variation of access concept (access, accessibility, availability, affordability, acceptability, accommodation, utilization, deprivation, disparity and equity) in connection with spatial terms (geographic information, GIS, geomapping, location-allocation, and spatial analysis) and dialysis (dialysis, haemodialysis, renal and kidney). To combine the search terms within each category, we utilized the disjunction $O R$, and to combine categories we utilized the conjunction $A N D$.

In order to identify additional relevant information related to ADS and GIS, the reference lists obtained were surveyed manually. To boost our search strategy further, we also looked for the so called gray literature, i.e. reports, standards, manuals and guidelines on the topic using general search engines such as Google. No date or study design limitation was imposed in any of the research steps described. The complete search strategy is available upon request.

\section{Eligibility criteria}

After the literature search had been completed, the EndNote X5 (Thomson Reuters, New York, NY, USA) reference management software was applied to aggregate all search returns. The articles were then screened and each study assessed independently for eligibility by two of the authors in different combinations. A study was considered eligible for inclusion if it included assessment of ADS as the primary or secondary outcome, while it was excluded if i) it had not clearly reported and calculated ADS indicators; ii) it lacked a methodological description of the measurement of ADS or its indicators; or iii) it consisted of a letter to the editor, an editorial, general comments, a position paper or it was an unstructured paper.

The full text of the qualified studies was read, tagged and summarized by one author and verified by one other author. A brief flow diagram of the strategy is depicted in Figure 1.

\section{Data extraction}

Studies deemed eligible for review underwent data extraction. For each paper, essential data items related to ADS measurement were extracted and fitted into a form with a choice of headings, such as ADS indicator; Factor(s) affecting the indicator; Method of measurement; Primary outcome measures; Secondary outcome measures; and Study design. Additional properties, such as conclusive comments and suggested measurement intervals, were also recorded when available and applicable. 


\section{Quality assessment}

Since systematic mapping reviews mainly aim to describe the state of the art of a particular topic, it is desirable to include communication types of a range as wide as possible. Due to the high diversity of study types in our review, this necessitated an informal quality assessment that was performed by classifying the literature by type of study. We used a mixed-approach scoring system as applied by Azizi et al. (2016) under similar circumstances (Azizi et al., 2016). Quality scores were assigned by two authors and verified by a third author. The weight of the literature was assigned according to their study design by the quality scoring system. In this approach, papers such as reviews and randomized controlled trials (RCTs) achieved the highest score (score 4) while score one was the lowest score. In this approach, the gray literature was given score one as formal or expert consensus regarding quality score. The summary of the quality assessment approach is outlined in Table 1.

\section{Data analysis}

Following mapping review methods, a tabular method for the synthesis of qualitative research findings was used. Also, the quantity and quality of the literature were characterized by study design and other key features, and need for some primary or secondary research was identified.

\section{Results}

Out of 1119 literature items collected, 76 were deemed eligible for further full-text review. After reviewing the full-text studies for final content match, 36 were selected for the review. Further details pertaining to the included studies are shown in Figure 1.

\section{Characteristics of included studies}

Three groups of studies were identified. The first group included eight cross-sectional studies addressing ADS and treating it as a primary outcome. The focus of these communications was the design of a GIS-based model intended for gauging the degree of ADS. They mostly calculated potential travel time (Roderick et al., 1999; Christie et al., 2005; White et al., 2006; Yang et al., 2006; Judge et al., 2012; Matsumoto et al., 2012; Stephens et al., 2013) or facility capacity (White et al., 2006; Yang et al., 2006; Matsumoto et al., 2012) as the key indicators of spatial accessibility. Some papers in this group also considered deprivation (Roderick et al., 1999; Christie et al., 2005; White et al., 2006; Judge et al., 2012) as an important, non-spatial indicator of ADS. One study designed a measure to calculate actual travel time revealed significant effects on the travel time of other non-spatial factors such as sex, income level, caregivers, transportation mode, education level, ethnicity and personal vehicle ownership. It demonstrated the large gap between potential travel time and actual travel time (Kiani et al., 2017). Another study showed that ignoring facility capacity and accounting only for travel time when evaluating ADS may result in misleading conclusions (Matsumoto et al., 2012). Further details pertaining to this group are outlined in Table 2 .

The second group of studies (10 peer-reviewed papers) considered gauging ADS as a secondary outcome, while their primary outcome measures focused on the association between ADS and health-related outcomes (Tonelli et al., 2007; Moist et al., 2008; Diamant et al., 2010; Rucker et al., 2011; Thompson et al., 2012;
Miller et al., 2014) determining the relationship between ADS and prevalence rates of dialysis patients (Kashima et al., 2012) or designing models to locate dialysis facilities (Ayyalasomayajula et al., 2011; Salgado et al., 2011; Faruque et al., 2012). Almost all of them calculated potential travel impedance as an indicator of ADS based on GIS.

Finally, the third group of 16 studies considered factors affecting ADS. Among them, were ten peer-reviewed articles (Smith et al., 1985; Tonelli et al., 2006; Hall et al., 2008; Prakash et al., 2010; Matsumoto et al., 2013; Omrani-Khoo et al., 2013; Rodriguez et al., 2013; Plantinga et al., 2014; Saunders et al., 2014; Kiani et al., 2017), one case series (Tshamba et al., 2014), one proceeding (Richard et al., 2009), and four gray literature items (Maheswaran et al., 2003; Mactier, 2007; Levinson, 2011; Amy Martin, 2013).

Considering the contents of the total number of GIS-based studies discussed here and the literature extracted from their comprehensive reference lists, 41 factors affecting ADS were determined (Table 3). Travel impedance, especially travel time, were the main indicators of spatial accessibility, the rest were mostly non-spatial. Some studies emphasize that factors such as ethnicity or the patient's health insurance status also affect ADS (Kashima et al., 2012; Thompson et al., 2012; Saunders et al., 2014), while other studies imply that they do not affect ADS (White et al., 2006; Matsumoto et al., 2012). The factors we found in our literature search and their frequency are given in Table 3.

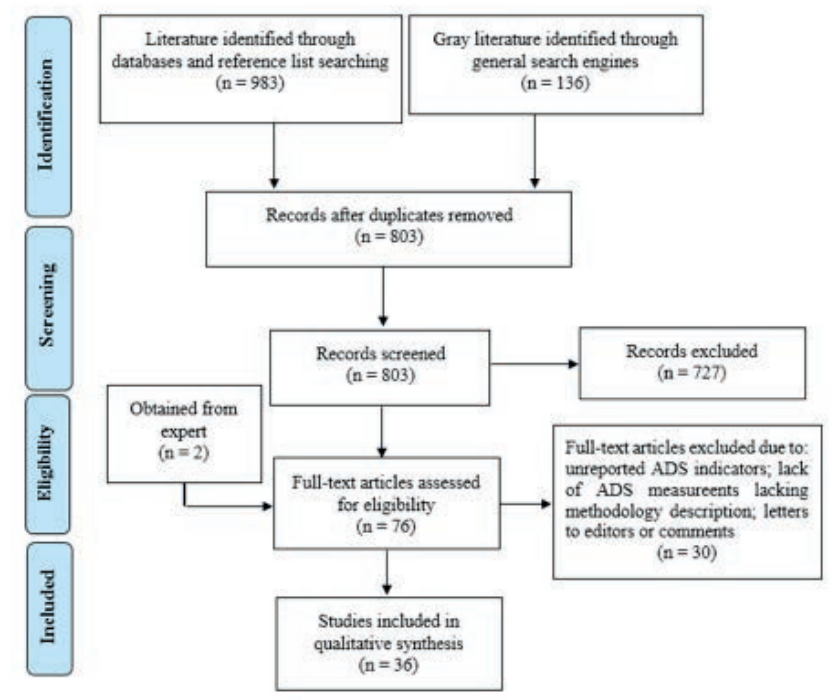

Figure 1. PRISMA Flowchart of included and excluded studies.

Table 1. Classification according to evidence quality.

\begin{tabular}{lc} 
Tlype of evidence & Score \\
RCT*, meta-analysis, systematic review & 4 \\
Case-control, cohort studies, quasy-experimental studies & 3 \\
\hline Observational studies (case reports, case series) & 2 \\
Formal/expert consensus & 1
\end{tabular}




\section{Discussion}

Our systematic mapping review of the evidence revealed that current GIS-based measures of ADS tend to calculate potential ADS instead of a realized one (Table 2). Also, we found no study

including both spatial and non-spatial dimensions of ADS into one framework that could produce a more realistic score than current attempts in this direction. However, in a recent study performed in Iran, Kiani et al. (2017) developed an integrated measure of ADS by calculating a reasonable measure of actual travel time in contrast to previous reports that mainly focus on estimated potential

Table 2. List of studies aiming at a GIS-based measure of access to dialysis service (ADS): an extraction table.

$\begin{array}{llll}\begin{array}{l}\text { Reference, Setting and study } \\ \text { country }\end{array} \text { participant } & \begin{array}{l}\text { Indicators } \\ \text { discussed }\end{array} & \text { Component employed }\end{array}$

Roderick et al., Renal facilities Multilevel modelling using

$1999 \quad$ Number of patients: 5,715 Poisson regression based

England, UK on patient postcodes matched

to each census ward

Dependent variable: the number

of accepted patients

(used as access indicator)

Independent effects modelled:

1) age and sex; 2) census ward need factors and supply factors; and 3) district health authority level effects
Travel impedance Spatial Service availability accessibility Jarman index ${ }^{\mathrm{a}} \quad$ Deprivation Townsend index ${ }^{\mathrm{b}}$ Carstairs index ${ }^{c}$ DoE Index ${ }^{d}$

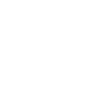

\begin{tabular}{|c|c|}
\hline $\begin{array}{l}\text { Christie et al., } \\
2005 \\
\text { Wales, UK }\end{array}$ & $\begin{array}{l}\text { Patient registers } \\
\text { and main and } \\
\text { satellite dialysis } \\
\text { facilities } \\
\text { Population: } 2.4 \text { million } \\
\text { Number of patients: } 1,514\end{array}$ \\
\hline $\begin{array}{l}\text { White et al., } \\
2006 \\
\text { Wales, UK } \\
\text { Wales }\end{array}$ & $\begin{array}{l}\text { Main dialysis facilities } \\
\text { Population: } 2.9 \text { million } \\
\text { Number of patients: } \\
2,173\left(\text { RRT }^{\mathrm{h}}\right) \text {, } \\
821\left(\mathrm{HD}^{\mathrm{j}}\right), 342 \text { (PDk) }\end{array}$ \\
\hline
\end{tabular}

Calculated a fifth of small area deprivation using the Townsend index, three bands of travel time using GIS ${ }^{g}$, RRTh ${ }^{\mathrm{h}}$ prevalence, and comparing patients and population accessibility between catchments, and prevalence in the population by a fifth of small area deprivation

UK Renal Registry,

Welsh population census data and WIMD 2005 analyzed using GISs patient location;

dialysis facilities geocoded and travel time calculated. Facility capacity calculated using the number of dialysis consoles assuming two shifts of dialysis per day and each patient received thrice a week. Linear regression analysis of data based on a $7 \%$ increase in the number of dialysis patients per year

$\begin{array}{ll}\text { Yang et al., } & \text { Dialysis facilities (44), } \\ 2006 & \text { census block data and } \\ \text { USA } & \text { dialysis consoles (867) } \\ & \text { Number of patients: } 3,722\end{array}$

Travel time, Spatial

Two GISg-based methods, Travel time, 2SFCAl and kernel density applied Facility considering the number of dialysis capacity Number of patients: 3,722 consoles in each dialysis facility presenting estimates of RRTh
Travel time, Spatial Townsend index accessibility Deprivation
All deprivation indices integrated as a customized index for best representing the deprivation component Emphasis on deprivation as the key indicator of both ADSe and NDS $S^{\mathrm{f}}$ but authors did not incorporate the components into one ADS framework Emphasis on impact of diabetes, hypertension and socio-economic factors on NDS ${ }^{f}$ but authors did not incorporate them in the model

Paper implies that age and gender affect ADSe and ND ${ }^{\mathrm{Sf}}$ and matches ADSe indicators for these factors

Travel time calculated as an indicator of spatial accessibility and deprivation calculated to compare RRTh prevalence between areas. The components were not incorporated into one ADSe framework Potential travel time calculated instead of actual travel time, and estimates of RRTh prevalence were lower in more deprived areas Facility capacity, accessibility WIMD $^{\mathrm{i}}$ Deprivation
Travel time and facility capacity calculated as indicators of spatial accessibility separately, and the WIMDi calculated to compare catchments prevalence It was estimated there would be 1,400 patients on haemodialysis in Wales by 2014

Emphasis on contributing factors led to some socioeconomic f actors being addressed in the WIMD ${ }^{\mathrm{i}}$ but some others, such as age, were not addressed as the supply and the number of patients as the demand with a 30-minute threshold for travel time. Demand estimate based on the population in each census tract multiplied by $0.12 \%$
Both methods integrated two indicators to calculate spatial accessibility. The implied revealed need most likely the same as the potential need when analyzing current ADS . The study implied that diabetes, hypertension, age, gender and race may influence the $\mathrm{ND}$ S $\mathrm{S}$ but this variable was not adjusted for them 
travel time (Christie et al., 2005; White et al., 2006; Yang et al., 2006; Judge et al., 2012; Matsumoto et al., 2012; Stephens et al., 2013) However, the results are still far from a truly realized ADS index since the key ADS indicator of facility capacity generally remains ignored.

Matsumoto et al. (2012) compared two GIS-based ADS mea- sures, the capacity-distance model and the distance model, and found the former to be more realistic than the latter. In the capacity-distance model, which addresses both travel time and facility capacity, patients were forced to travel further due to capacity limitations at closer centres. Furthermore, an American study noted that proximity to dialysis services does not directly translate into

Table 2. Continued from previous page.

$\begin{array}{ll}\text { Reference, Setting and study Method employed } \\ \text { country } & \text { participant }\end{array}$

$\begin{array}{ll}\text { Matsumoto } & \text { Dialysis facilities (98) } \\ \text { et al., } & \text { Number of patients: } 7,374 \\ 2012 & \end{array}$

2012

Japan
Travel time calculated by

GISs in two models: the distance model (travel time to nearest facility) and the capacity-distance model (both travel time and facility capacity). The latter allowed facilities to accept the nearest patient (based on travel time) until capacity is full and then remit patients to the second-nearest facility run through facilities until all patients are accepted

\begin{tabular}{ll}
$\begin{array}{l}\text { Judge } \\
\text { et al., }\end{array}$ & Renal Registry and \\
2012 & England, UK \\
UK & $\begin{array}{l}\text { local authority } \\
\text { districts (354) }\end{array}$ \\
& \\
& \\
\hline Stephens & Centers for Medicare \\
et al., & and \\
2013 & Medicaid services \\
USA & (5,007) \\
& Number of patients: \\
& 332,117
\end{tabular}

$\begin{array}{ll}\text { Kiani } & \text { Dialysis facilities (6) } \\ \text { et al., } & \text { Number of patients: } 168 \\ 2017 & \\ \text { Iran } & \end{array}$

Multilevel Poisson regression models constructed separately for incidence and prevalence. Travel times and dialysis facilities small area estimates of RRTh prevalence produced for all 354 local districts. Three data points estimated: (1) patients' location; currently serving patient; and (3) location of replacement facility. GISg-based calculation of travel impedance between them Included calculations: 1. Travel impedance from patient location to current facility; 2. Travel impedance from patient location to replacement facility; 3. Incremental travel impedance for travel to replacement facility compared with travel

Data for AAT ${ }^{\mathrm{n}}$ and other spatial or non-spatial variables gathered via a semi-structured questionnaire for patients.

\section{Indicators Comment} discussed catchment areas estimated by GISg; (2) location of dialysis facility to current facility Univariate analysis and univariate general linear model used to identify $\mathrm{AAT}^{\mathrm{n}}$ associated factors Driving time and distance calculated using Google maps; AAT of rural and urban analyzed separately
Travel time, Facility accessibility capacity Spatial
accessibility
Spatial IMDm accessibility Deprivation
The distance model addresses only travel time to calculate spatial accessibility, while the capacity-distance model uses two ADSe indicators jointly to calculate spatial accessibility Potential travel time calculated instead of real travel time; no model considering non-spatial factors indicating a potential gap between calculated accessibility and real access, which is more than just the equity of travel time

Components not integrated into one ADSe framework Deprivation adjusted for socio-demographic differences but without adjusting is directly relative to $\mathrm{NDS}^{\mathrm{f}}$ Potential travel time calculated instead of real travel time

\begin{tabular}{|c|c|c|}
\hline Travel time & $\begin{array}{l}\text { Spatial } \\
\text { accessibility }\end{array}$ & $\begin{array}{l}\text { Actual data not obtained from each } \\
\text { patient's original dialysis facility. } \\
\text { As it was assumed that patients } \\
\text { utilize the closest location, potential } \\
\text { accessibility was calculated } \\
\text { Indication that facility capacity is } \\
\text { a key indicator of spatial accessibility } \\
\text { to dialysis facilities, } \\
\text { but calculation of spatial accessibility } \\
\text { not integrated with travel time. } \\
\text { Non-spatial factors emphasized } \\
\text { although not integrated with } \\
\text { the spatial accessibility component } \\
\text { to calculate a more realistic ADSe }\end{array}$ \\
\hline
\end{tabular}

Travel Realized spatial Non-spatial factors (sex, income impedance accessibility level, caregivers, transportation mode, education level, ethnicity and personal vehicle ownership) influenced the revealed access identified in rural and urban groups but spatial factors were identified only in the former AAT $^{\mathrm{n}}$ calculated but some other important $\mathrm{ADS}$ indicators such as facility capacity was ignored

aA scoring system developed by the British general practitioner Brian Jarman (b. 1933) for the level of social deprivation; ba measure of material deprivation within a population; ${ }^{c}$ an index of deprivation used in spatial epidemiology to identify socio-economic confounding; dan index of urban poverty published by the UK Department for Environment, Food and Rural Affairs designed to assess relative levels of deprivation in local

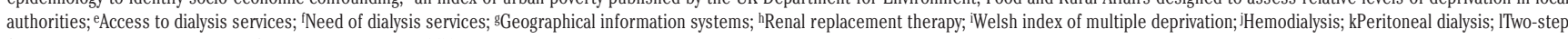
floating catchment area; mIndex of multiple deprivation; ${ }^{\mathrm{B} A A T}$ actual access time. 
access owing to potential racial segregation (Saunders et al., 2014). However, both these studies calculated potential travel time. Meanwhile, many British studies (Roderick et al., 1999; Christie et al., 2005; White et al., 2006; Judge et al., 2012) emphasize the importance of non-spatial factors, e.g., Kiani's et al. study (2017), considering deprivation as one of the main ADS indicators. Indeed, controversies in this field show a gap between calculated and real ADS. Although this study did not find any research integrating spatial and non-spatial factors into a GIS-embedded model for measuring ADS, various GIS-oriented studies join some spatial ADS indicators together to measure spatial accessibility (Yang et al., 2006; Matsumoto et al., 2012). For instance, Yang et al. compared two GIS-based methods, the 2SFCA approach and the kernel density method, in a case study on renal dialysis facilities in Chicago, USA. In this study, based on the main spatial ADS indicators travel time and facility capacity, the 2SFCA method produced better accessibility ratios overall (Yang et al., 2006). Although, this work only integrated spatial ADS dimensions, it has provided a platform that has been successfully used in other contexts, especially in primary healthcare where an overall measure including both spatial and non-spatial factors of access is included (Wang and Luo, 2005; Bagheri et al., 2008; McGrail and Humphreys, 2009; McGrail and Humphreys, 2015). For example, Bagheri et al. (2008) developed an integrated access to primary healthcare (APH) index which combined spatial accessibility, calculated by 2 SFCA method, and a need index into one framework, while McGrail and Humphreys $(2009,2015)$ improved the 2SFCA method by introducing a concept based on three key components, i.e. spatial accessibility, population health needs and mobility.

The progress covered by this review, as well as the gaps revealed, raises the hypothesis that an integrated ADS index should calculate access more realistically than current GIS-based measures. However, further research examining whether this hypothesis is correct or not is needed. This mapping review provides some evidence-based recommendations that may help researchers and policymakers perform a primary study assessing this hypothesis. Three components of the 2SFCA platform should be addressed in order to construct an integrated ADS index, i.e. spatial accessibility, mobility and NDS.

Spatial accessibility must take into account both accessibility and availability. Travel time, discussed by most current studies, should be calculated as an indicator of accessibility with a threshold of 30 minutes as haemodialysis guidelines recommended (Mactier, 2007). However, actual travel time as proposed by Kiani et al. (Kiani et al., 2017) should be used together with facility capacity, expressed as the number of dialysis machines (supply) to the number of patients (demand) in each facility (Yang et al., 2006; Matsumoto et al., 2012) that seems to be the key availability indicator. It is as important as the travel time and should be incorporated into the 2SFCA framework with an appropriate threshold to construct catchment areas more realistically. Patients need dialysis thrice a week according to current haemodialysis guidelines (Mactier, 2007), which means that each machine can serve up to four patients per week (two patients on even days and two patients on odd days) leading to a supply-to-demand ratio threshold of $1 / 4$. If, regardless of this threshold, all catchments are constructed with a radius of 30 minutes travel time, some of them might include patients more than their facility capacity.

Mobility is defined as the population's ability to overcome distance (Bisht et al., 2010). Taking the relative population size of those aged either $<18$ years or $>75$ years as the measure of reduced personal mobility, McGrail et al. (McGrail and Humphreys, 2009)

Table 3. Factors affecting access to dialysis services elicited from literature.

\begin{tabular}{|c|c|c|c|}
\hline Factors/Variables & Rate & Factors/Variables & Rate \\
\hline Patient age & 14 & Population density & 2 \\
\hline Patient gender & 11 & Province need & 2 \\
\hline Patient ethnicity & 13 & Nephrology beds & 3 \\
\hline Patient's education status & 6 & Location of the patient's nephrologist & 1 \\
\hline Marital status & 1 & Patient's familiarity with facility & 1 \\
\hline Patient's income & 9 & Routine procedures at the facility & 1 \\
\hline Patient's employment status & 5 & Availability of transportation & 3 \\
\hline Patient's disability & 1 & Car ownership & 23 \\
\hline Patient mobility & 4 & Road quality linking home and facility & 4 \\
\hline Patient's comorbidities & 1 & Transportation mode & 3 \\
\hline Patient's health insurance status & 7 & Travel cost & 17 \\
\hline Home owner & 1 & Travel speed & 1 \\
\hline Socioeconomic status of neighbourhood & 1 & Travel time & 3 \\
\hline Location of dialysis patient's residence & 12 & Travel distance & 1 \\
\hline Language status & 1 & Time of the day for dilalysis visit & 1 \\
\hline Number of dialysis facilities & 2 & Degree of family support & 3 \\
\hline Facility type (satellite or in-center) & 4 & Public assistance & 1 \\
\hline Dialysis type needed* & 2 & Availability of human resources & 1 \\
\hline Facility capacity & 10 & Deprivation & 10 \\
\hline Number of dialysis consoles per facility & 9 & Climate conditions & 1 \\
\hline Cost of care & 2 & & \\
\hline
\end{tabular}


point out that the three indicators, i.e. households without a car, individuals with reduced mobility and public transport availability, measure different aspects of mobility and that correlations between them are small. Considering this nature of mobility, it seems useful to apply these three indicators when calculating mobility in the ADS context.

Revealed NDS (the actual demand) is most likely to be the same as the potential NDS when the current ADS is calculated (Yang et al., 2006), but future ADS calculations will differ because the number of patients then is unclear, in particular as the prevalence of end-stage renal disease (ESRD) is increasing (Chadban et al., 2003; Eggers, 2011). An easy way to estimate NDS is by multiplying the annual growth rate of demand at current demand (White et al., 2006; Yang et al., 2006). But an adjustment is needed if there is an increase in diabetes which is already anticipated (Roderick et al., 1999; Yang et al., 2006) and hypertension (Roderick et al., 1999; Yang et al., 2006), the two principal causes of ESRD. In addition, deprivation (Roderick et al., 1999; Thomas, 2005; Judge et al., 2012), age (Roderick et al., 1999; Thomas, 2005), gender (Roderick et al., 1999; Yang et al., 2006) and ethnicity (Roderick et al., 1996; Judge et al., 2012) may influence the NDS differently in the future.

To our knowledge, this is the first systematic mapping review of the available literature aimed at identifying gaps in current GISbased ADS measures and developing evidence-based recommendations. However, by limiting the search strategy by specifying it for the GIS category and developing a list of factors affecting ADS elicited, we may have lost evidence in studies exclusively focused on exploring factors affecting ADS. This would be true, even though we had a comprehensive list referencing the literature elicited from our systematic mapping review. However, this was secondary outcome measure of the study, and we suggest a systematic review with a wider scope that could list all factors affecting ADF. Although we did not systematically focus on seeking factors affecting NDS, we think that our findings related to estimating NDS are appropriate and enough. Moreover, we found that hospitalization rate (Rucker et al., 2011) and mortality rate (Rucker et al., 2011; Thompson et al., 2012) are negatively associated with ADS, facts that can be used for validation of the integrated index of ADS, a proper validation of this index remains open for future study. Finally, even though we highlighted the absence of evidence-based recommendations incorporating indicators related to the acceptability dimension in an integrated ADS index, we could not alleviate this weakness of current GIS-based measures of ADS, which needs further research.

\section{Conclusions}

Current GIS-based measures of ADS tend to calculate potential ADS instead of a realized one and there is a need to examine whether an integrated index of ADS can calculate a realistic score. Listed factors affecting ADS are mainly non-spatial encouraging the design of an integrated ADS index produce better ADS score than those currently advocated. The mapping review strongly suggests exploring the hypothesis that a combined index of ADS including most dimensions of ADS can be developed and produce a better ADS score than current available. The 2SFCA method may be capable of providing a platform for this aim as the study recommended and researchers and policymakers are encouraged to examine and validate this hypothesis.

\section{References}

Amy Martin KJB, Nathan Hale, Janice C. Probst, 2013. Dialysis availability in rural America, South Carolina Rural Health Research Center.

Andersen RM, 1995. Revisiting the behavioral model and access to medical care: does it matter? J Health Soc Behav 36:1-10.

Ayyalasomayajula B, Wiebe N, Hemmelgarn BR, Bello A, Manns B, Klarenbach S, Tonelli M, 2011. A novel technique to optimize facility locations of new nephrology services for remote areas. Clin J Am Soc Nephrol 6:2157-64.

Azizi A, Aboutorabi R, Mazloum-Khorasani Z, Hoseini B, Tara M, 2016. Diabetic personal health record: a systematic review article. Iran J Publ Health 45:1388-98.

Bagheri N, Benwell GL, PGDipSci M, 2008. Modelling Accessibility to primary health care using a spatial accessibility index and a need index. Hawai 'I J Pub Health 1:14.

Bisht SS, Mishra V, Fuloria S, 2010. Measuring accessibility for inclusive development: a census based index. Soc Indicators Res 98:167-81.

Chadban SJ, Briganti EM, Kerr PG, Dunstan DW, Welborn TA, Zimmet PZ, Atkins RC, 2003. Prevalence of kidney damage in Australian adults: The AusDiab kidney study. J Am Soc Nephrol 14:S131-8.

Christie S, Morgan G, Heaven M, Sandifer Q, Woerden H, 2005. Analysis of renal service provision in south and mid Wales. Publ Health 119:738-42.

Diamant MJ, Harwood L, Movva S, Wilson B, Stitt L, Lindsay RM, Moist LM, 2010. A comparison of quality of life and travel-related factors between in-center and satellite-based hemodialysis patients. Clin J Am Soc Nephrol 5:268-74.

Eggers PW, 2011. Has the incidence of end-stage renal disease in the USA and other countries stabilized? Curr Opin Nephrol Hypertens 20:241-5.

Faruque LI, Ayyalasomayajula B, Pelletier R, Klarenbach S, Hemmelgarn BR, Tonelli M, 2012. Spatial analysis to locate new clinics for diabetic kidney patients in the underserved communities in Alberta. Nephrol Dial Transplant 27:4102-9.

Grant MJ, Booth A, 2009. A typology of reviews: an analysis of 14 review types and associated methodologies. Health Info Libr J 26:91-108.

Guagliardo MF, 2004. Spatial accessibility of primary care: concepts, methods and challenges. Int J Health Geogr 3:3.

Hall YN, O'Hare AM, Young BA, Boyko EJ, Chertow GM, 2008. Neighborhood poverty and kidney transplantation among US asians and pacific islanders with end-stage renal disease. Am J Transplant 8:2402-9.

Judge A, Caskey FJ, Welton NJ, Ansell D, Tomson CR, Roderick PJ, Ben-Shlomo Y, 2012. Inequalities in rates of renal replacement therapy in England: does it matter who you are or where you live? Nephrol Dial Transplant 27:1598-607.

Kashima S, Matsumoto M, Ogawa T, Eboshida A, Takeuchi K, 2012. The impact of travel time on geographic distribution of dialysis patients. PLoS One 7:e47753.

Kiani B, Bagheri N, Tara A, Hoseini B, Tabesh H, Tara M, 2017. Revealed access to haemodialysis facilities in northeastern Iran: Factors that matter in rural and urban areas. Geospat Health 12:237-44.

Kiani B, Bagheri N, Tara A, Hoseini B, Tara M, 2017. Haemodialysis services in the northeastern region of Iran. Geospat Health 12:130-6. 
Levesque JF, Harris MF, Russell G, 2013. Patient-centred access to health care: conceptualising access at the interface of health systems and populations. Int J Equity Health 12:18.

Levinson DR, 2011. Access to kidney dialysis services at indian health service and tribal facilities. Department of Health and Human Services USA.

Mactier R, 2007. Clinical practice guidelines module 2: haemodialysis. UK Renal Association.

Maheswaran R, Payne N, Meechan D, Burden RP, Fryers PR, Wight J, Hutchinson A, 2003. Socioeconomic deprivation, travel distance, and renal replacement therapy in the Trent Region, United Kingdom 2000: an ecological study. J Epidemiol Commun Health 57:523-4.

Mao L, Nekorchuk D, 2013. Measuring spatial accessibility to healthcare for populations with multiple transportation modes. Health Place 24:115-22.

Matsumoto M, Kashima S, Ogawa T, Takeuchi K, 2013. Do rural and remote areas really have limited accessibility to health care? Geographic analysis of dialysis patients in Hiroshima, Japan. Rural Remote Health 13:2507.

Matsumoto M, Ogawa T, Kashima S, Takeuchi K, 2012. The impact of rural hospital closures on equity of commuting time for haemodialysis patients: simulation analysis using the capacitydistance model. Int J Health Geogr 11:28.

McGrail MR, Humphreys JS, 2009. The index of rural access: an innovative integrated approach for measuring primary care access. BMC Health Serv Res 9:124.

McGrail MR, Humphreys JS, 2015. Spatial access disparities to primary health care in rural and remote Australia. Geospat Health 10:358.

McLafferty SL, 2003. GIS and health care. Annu Rev Public Health 24:25-42.

Miller LM, Vercaigne LM, Moist L, Lok CE, Tangri N, Komenda P, Rigatto C, Mojica J, Sood MM, 2014. The association between geographic proximity to a dialysis facility and use of dialysis catheters. BMC Nephrol 15:40.

Moist LM, Bragg-Gresham JL, Pisoni RL, Saran R, Akiba T, Jacobson SH, Fukuhara S, Mapes DL, Rayner HC, Saito A, Port FK, 2008. Travel time to dialysis as a predictor of health-related quality of life, adherence, and mortality: the Dialysis Outcomes and Practice Patterns Study (DOPPS). Am J Kidney Dis 51:64150 .

Omrani-Khoo H, Lotfi F, Safari H, Zargar Balaye Jame S, Moghri J, Shafii M, 2013. Equity in distribution of health care resources; assessment of need and access, using three practical indicators. Iran J Public Health 42:1299-308.

Plantinga LC, Kim M, Goetz M, Kleinbaum DG, McClellan W, Patzer RE, 2014. Pre-end-stage renal disease care not associated with dialysis facility neighborhood poverty in the United States. Am J Nephrol 39, 50-8.

Prakash S, Rodriguez RA, Austin PC, Saskin R, Fernandez A, Moist LM, O'Hare AM, 2010. Racial composition of residential areas associates with access to pre-ESRD nephrology care. J Am Soc Nephrol 21:1192-9.

Richard JB, Aldigier JC, Le Mignot L, Glaudet F, Ben Said M, Landais P, 2009. Equity of accessibility to dialysis facilities. Stud Health Technol Inform 150:777-81.

Roderick P, Clements S, Stone N, Martin D, Diamond I, 1999. What determines geographical variation in rates of acceptance onto renal replacement therapy in England? J Health Serv Res Policy 4:139-46.
Roderick PJ, Raleigh VS, Hallam L, Mallick NP, 1996. The need and demand for renal replacement therapy in ethnic minorities in England. J Epidemiol Commun Health 50:334-9.

Rodriguez RA, Hotchkiss JR, O'Hare AM, 2013. Geographic information systems and chronic kidney disease: racial disparities, rural residence and forecasting. J Nephrol 26:3-15.

Rucker D, Hemmelgarn BR, Lin M, Manns BJ, Klarenbach SW, Ayyalasomayajula B, James MT, Bello A, Gordon D, Jindal KK, Tonelli M, 2011. Quality of care and mortality are worse in chronic kidney disease patients living in remote areas. Kidney Int 79:210-7.

Russell DJ, Humphreys JS, Ward B, Chisholm M, Buykx P, McGrail M, Wakerman J, 2013. Helping policy-makers address rural health access problems. Aust J Rural Health 21:61-71.

Salgado TM, Moles R, Benrimoj SI, Fernandez-Llimos F, 2011. Designing a model to minimize inequities in hemodialysis facilities distribution. Geospat Health 6:5-12.

Saunders MR, Lee H, Maene C, Schuble T, Cagney KA, 2014. Proximity does not equal access: racial disparities in access to high quality dialysis facilities. J Racial Ethn Health Disparities 1:291-9.

Saurman E, 2016. Improving access: modifying Penchansky and Thomas's theory of access. J Health Serv Res Policy 21:36-9.

Smith MD, Robson AM, Woodward RS, Michelman JE, Valerius TJ, Hong BA, 1985. Geographic access to health care services: the case of maintenance hemodialysis. Am J Kidney Dis 5:19-26.

Stephens JM, Brotherton S, Dunning SC, Emerson LC, Gilbertson DT, Harrison DJ, Kochevar JJ, McClellan AC, McClellan WM, Wan S, Gitlin M, 2013. Geographic disparities in patient travel for dialysis in the United States. J Rural Health 29:339-48.

Thomas M, 2005. Deprivation and dialysis: pathways to kidney failure in Australian Aborigines. Adv Chronic Kidney Dis 12:84-7.

Thompson S, Gill J, Wang X, Padwal R, Pelletier R, Bello A, Klarenbach S, Tonelli M, 2012. Higher mortality among remote compared to rural or urban dwelling hemodialysis patients in the United States. Kidney Int 82:352-9.

Tonelli M, Hemmelgarn B, Kim AK, Bertazzon S, Klarenbach S, Manns B, Wiebe N, Culleton B, Gill JS, 2006. Association between residence location and likelihood of kidney transplantation in Aboriginal patients treated with dialysis in Canada. Kidney Int 70:924-30.

Tonelli M, Manns B, Culleton B, Klarenbach S, Hemmelgarn B, Wiebe N, Gill JS, 2007. Association between proximity to the attending nephrologist and mortality among patients receiving hemodialysis. Cmaj 177:1039-44.

Tshamba HM, Van Caillie D, Nawej FN, Kapend FM, Kaj FM, Yav GD, Nawej PT, 2014. Risk of death and the economic accessibility at the dialysis therapy for the renal insufficient patients in Lubumbashi city, Democratic Republic of Congo. Pan Afr Med J 19:61.

Wang F, Luo W, 2005. Assessing spatial and nonspatial factors for healthcare access: towards an integrated approach to defining health professional shortage areas. Health Place 11:131-46.

White P, James V, Ansell D, Lodhi V, Donovan KL, 2006. Equity of access to dialysis facilities in Wales. Qjm 99:445-52.

Yang DH, Goerge R, Mullner R, 2006. Comparing GIS-based methods of measuring spatial accessibility to health services. J Med Syst 30:23-32. 